\title{
Loss of function mutation in ELF4 causes autoinflammatory and immunodeficiency disease in human
}

Gan Sun ${ }^{1,2,3}$, Luyao Qiu ${ }^{1,2,3}$, Yunfei An $^{1,2,3,4}$, Yuan Ding ${ }^{1,2,3}$, Lina Zhou ${ }^{1,2,3}$, Junfeng

$\mathrm{Wu}^{1,2,3,4}$, Xuemei $\mathrm{Tang}^{1,2,3,4}$, Huawei $\mathrm{Xia}^{5}$, Lili $\mathrm{CaO}^{5}$, Fuping You ${ }^{5}$, Xiaodong Zhao ${ }^{1,2,3,6}$, Hongqiang $\mathrm{Du}^{1,2,3,4 *}$

1. National Clinical Research Center for Child Health and Disorders, Children's Hospital of Chongqing Medical University, Chongqing, China.

2. Ministry of Education Key Laboratory of Child Development and Disorders, Children's Hospital of Chongqing Medical University, Chongqing, China.

3. Chongqing Key Laboratory of Child Infection and Immunity, Children's Hospital of Chongqing Medical University, Chongqing, China.

4. Department of Rheumatology \& Immunology, Children's Hospital of Chongqing Medical University, Chongqing, China.

5. Institute of Systems Biomedicine, Department of Immunology, School of Basic

Medical Sciences, Beijing Key Laboratory of Tumor Systems Biology, Peking

University Health Science Center, Beijing 100000, China

6.The Second affiliated Hospital of Chongqing Medical University, Chongqing 400014, China

* Correspondence

Corresponding authors: Hongqiang Du (ㅎoctordo@aliyun.com) and Xiaodong Zhao (zhaoxd530@aliyun.com)

\begin{abstract}
Monogenic autoinflammatory diseases (mAIDs) are a heterogeneous group of diseases affecting primarily innate immunity, with various specific genetic causes. Genetic diagnosis of mAIDs can assist in the patient's management and therapy. However, a large number of sporadic and familial cases remain genetically
\end{abstract}


uncharacterized. Here, we described a pediatric patient suffering from recurrent viral and bacterial respiratory infection, refractory oral ulcer and constipation, who was clinically diagnosed of inborn errors of immunity (IEI). In an effort to establish genetic diagnosis, no known causative genes were identified by whole-exome sequencing. However, manually going through bioinformatically predicted candidate genes, we suspected and prioritized ELF4 (chrX:129205133 A>G, c.691T>C, p.W231R) as the genetic cause for our patient. We then evaluated the pathogenicity of this mutation by both various bioinformatic methods and preliminary but definitive experimental approach. Our data suggested that W231R mutant ELF4 is a "loss of function" mutation causing decreased protein stability and decreased trans-activation activity. Thus, we identified a novel mAID, which we termed "X-linked autoinflammatory and immunodeficiency disease associated with ELF4, X-AIDE”.

Key Words: ELF4; autoinflammatory disease; immunodeficiency; inborn errors of immunity; loss of function

\section{Introduction}

Monogenic autoinflammatory diseases (mAIDs) are a heterogeneous subgroup of inborn errors of immunity (IEI) diseases or primary immunodeficiency diseases (PIDs), which are marked by hyperactivation of the innate immune system without apparent involvement of either autoantibodies or autoreactive $\mathrm{T}$ cells ${ }^{1}$. The autoinflammatory disease spectrum has expanded rapidly to include more than 40 distinct monogenic conditions, which can be roughly categorized into "Inflammasomopathies and other diseases arising through IL-1-family cytokines", “Type I Interferonopathies”, "Disorders of NF-kB and/or aberrant TNF activity”, and "Autoinflammation mediated by other mechanisms",2. Across these categories, mAIDs vary widely in their presentation and clinical manifestations. While the definitive diagnosis is supported by genetic testing, in many practical settings, the diagnosis remains clinical $^{3}$. Even in the monogenic diseases a clinical approach is first 
necessary to narrow the genetic search for the correct disease ${ }^{4}$. mAIDs should be suspected in patients with recurrent fever unexplained by infections and/or with episodic stereotypic symptoms in various organs and tissues, especially the skin, gastrointestinal tract, musculoskeletal system, eyes, mucous membranes and central nervous system. When a clinical diagnosis of mAID is made, a genetic approach should be taken ${ }^{5}$, preferably such as the next-generation whole exome sequencing (WES) if applicable, which has fueled the discovery of novel genes in the past several years.

By definition, mAIDs do not involve adaptive immune responses, however, this simplistic division is at best a first approximation. Innate and adaptive immunity are closely interconnected, and dysfunction in one often disturbs function in the other ${ }^{2}$. For example, some diseases now considered autoinflammatory feature autoantibodies. In some of the newly described diseases, recurrent infections, indicative of an immunodeficiency, are seen along with autoinflammatory-related symptoms ${ }^{6}$. Thus, a more accurate understanding of the pathogenesis of mAIDs may need a thorough investigation of both innate and adaptive immunity.

ELF4 belongs to the E-Twenty-Six (ETS) domain transcription factor family and is involved in a variety of biological processes, including adaptive immune response ${ }^{7}$. Previously, we have shown that ELF4 is a critical transcription factor for the host antiviral response, during which ELF4 cooperates with nuclear factor-kB (NF- $\kappa B)$ to induce robust interferons and inflammatory cytokine production ${ }^{8}$. In addition, we recently showed Elf4-deficient mice are highly susceptible to DSS-induced colitis, a subgroup of inflammatory bowel disease ${ }^{9}$. The potential role of ELF4 in the pathogenesis of "Type I Interferonopathies" or "Disorders of NF- $\kappa B$ and/or aberrant TNF activity" has not been revealed in human.

Here we studied a Chinese family with a X chromosome-linked disorder demonstrating as recurrent viral and bacterial respiratory infection, refractory oral ulcer and constipation. Whole-exome sequencing on the proband and his parents was negative for any known causative genes. However, manually going through bioinformatically predicted candidate genes, we suspected and prioritized ELF4 
(chrX:129205133 A>G, c.691T>C, p.W231R), which was Sanger sequencing confirmed first, as the genetic cause for our patient. We then evaluated the pathogenicity of this mutation by both various bioinformatic methods and preliminary but robust experimental approach. Our data suggested that W231R mutant ELF4 is a "loss of function" mutation causing decreased protein stability and decreased trans-activation activity. Thus, we identified a novel mAID, for which we proposed the term "X-linked autoinflammatory and immunodeficiency disease associated with ELF4, X-AIDE".

\section{Results}

\section{Case characteristic summary}

The patient $(\mathrm{P} 1)$ presented with frequent upper and lower respiratory tract (URT) infection with blood routine test mostly showing normal WBC but increased lymphocyte percentage, which indicated a viral infection. He also suffers from recurrent and refractory oral ulcer and refractory constipation but no hematochezia, melena, abdominal pain, fever of unknown nor diarrhea. Regular IVIG infusion at a supportive dose ranging from $400 \mathrm{mg} / \mathrm{kg}$ to $1 \mathrm{~g} / \mathrm{kg}$ was advised and strictly followed by the patient. Frequency of respiratory infection dropped dramatically, however, oral ulcer still bothers him at a slightly lower frequency. The patient is at school age now. (Detailed medical history is available from the corresponding authors upon reasonable request).

\section{Genetic findings}

Upon suspecting PID, we performed whole-exome sequencing of whole blood sample from the proband and his parents. A hemizygous ELF4 mutation (chrX:129205133 A $>$ G, c.691T $>$ C, p.W231R) drew our attention. Sanger sequencing confirmed this (Fig. 1A), which is consistent with their phenotype. Firstly, this mutation does not occur in the $1000 \mathrm{~g}$ database, ExAC database and in-house database. This amino acid 
site is extremely conserved among the species (GERP++ score 5.45, classified as "conserved") and among ETS domain transcription factor super family members (Fig. 1B). Pathogenicity prediction using multiple methods classified this mutation as "Damaging", "Probably damaging", "Disease causing", "Pathogenic" or "Functional Impact High" (Table 2) ${ }^{11}$.

Secondly, ELF4 is a transcription factor and plays critical roles in both innate and adaptive immune responses ${ }^{8}$. This mutation site locates at the ETS domain of ELF4 (Fig. 1C), which is critical for its DNA binding ${ }^{13}$.

Thirdly, when we test the significance of highly conserved amino acid sites in the ETS domain of ELF4 in the induction of type I interferons (Fig. 1D), we found site 231 is among those essential for type I interferon induction (Fig. 1D). Taken together, we speculated that ELF4 mutation is the genetic cause for this undefined PID.

\section{W231R is a "loss of function" mutation}

To explore the more specific effects of this mutation, we first examined the stability change of the mutant ELF4 using DynaMut, a widely cited web-based tool for analysis and prediction of protein stability changes upon mutation using Normal Mode Analysis ${ }^{13}$. Because there is no experimental crystal structure data for both human and mouse ELF4 protein, we here used AlphaFold2-predicted structure data in PDB format ${ }^{14}$. The predicted $\Delta \Delta \mathrm{G}$ is $-1.380 \mathrm{kcal} / \mathrm{mol}$, classified as "Destabilizing". Structurally, various atomic contacts and bonds are predicted to be disrupted by the mutation (Fig. 2A). The $\Delta$ vibrational entropy energy between wild-type and mutant indicated increase of molecule flexibility $\left(\Delta \Delta \mathrm{S}_{\mathrm{Vib}}\right.$ ENCoM: $\left.1.215 \mathrm{kcal} . \mathrm{mol}^{-1} \cdot \mathrm{K}^{-1}\right)(\mathrm{Fig}$. 2B). Consistently, we found decreased expression of mutant ELF4 in patient's PBMC (Fig. 2C). To further confirm this, we constructed wild type and mutant ELF4 expression plasmids and performed Cycloheximide Chase Assay in HEK293T cells. Mutant ELF4 showed decreased stability compared to WT ELF4 (Fig. 2D).

Next, we explored the intrinsic functional impact of this mutation first using MutationAssessor, a widely used tool for prediction of functional impact of 
amino-acid substitutions in proteins ${ }^{15}$. The Functional Impact score is 3.55 indicating "highly functionally affected" (Table 2). We further tested this in our type I interferon luciferase reporter system and showed that WT ELF4 (human and mouse) can activate IFN- $\beta$ and IFN- $\alpha$ reporter in a dose-dependent manner while mutant ELF4 showed almost abolished trans-activation activity (Fig. 2E-2G). Collectively, our data suggested that W231R mutant ELF4 is a "loss of function" mutation in two senses, decreased protein stability and decreased trans-activation activity (possibly for decreased DNA binding activity).

\section{Discussion}

Autoinflammatory diseases were first recognized over two decades ago as distinct clinical and immunological entities caused by dysregulation in the innate immune system $^{16}$. Since then, advances in genomic techniques have made reaching a genetic diagnosis easier and led to the identification of new monogenic disorders. We here identified a novel mAID, which we termed "X-linked autoinflammatory and immunodeficiency disease associated with ELF4, X-AIDE”.

As pediatric immunologist, our diagnosis of a disease for a patient is firstly based on clinical manifestations and medical history of the patients and begins with a clinical suspicion or clinical diagnosis. For the diagnosis of mAIDs, a clinical suspicion should be made when: 1) Evidence for systemic inflammation (elevated acute phase reactants during disease flare e.g. C-reactive protein (CRP), erythrocyte sedimentation rate (ESR) and serum amyloid A (SAA). In some diseases, patients do not have strong evidence for systemic inflammation but may have organ or tissue specific inflammation (e. g. autoinflammatory disorders with prominent cutaneous manifestations where inflammation can be confirmed by skin biopsy). 2) The disease is likely to be inherited as a monogenic trait (early-onset symptoms in sporadic patients or multiple affected siblings or dominantly inherited symptoms). 3) Plausible candidate causal gene ${ }^{3}$. Our patient suffered from refractory oral ulcer with early onset and prolonged course. This local vasculitis is similar to what commonly seen in 
patients with Behçet's disease or with Haploinsufficiency of A20 (HA20) ${ }^{17}$, which indicates this disease may belong to the category of "Disorders of NF- $\kappa B$ and/or aberrant TNF activity". In addition, our patient also suffers from recurrent viral and bacterial respiratory infection with early onset and prolonged course, which further encourage us to made the diagnosis of primary immunodeficiency.

In some recently described novel mAIDs, autoinflammatory disease causing mutations also lead to defective immune responses and predispose to infection. Mutations in PLCG2 cause autoinflammation with antibody deficiency ${ }^{18}$. Deficiency of components of the linear ubiquitin chain assembly complex (LUBAC) leads to severe autoinflammation with immunodeficiency in association with polyglucosan myopathy ${ }^{19}$. Mutations in TRNT1 produce a syndrome of siderblastic anemia with B cell immunodeficiency, periodic fevers and developmental delay ${ }^{20}$. Patients with deficiency in WDR1 manifest autoinflammatory periodic fever, immunodeficiency and thrombocytopenia ${ }^{21}$. One potential explanation is that cell-type specific effects of mutated proteins lead directly to the combination of reduced immunity and enhanced inflammation. We previously showed ELF4 is critical for type I interferon induction during viral and bacterial infection ${ }^{8}$. Our patient harbors W231R mutation in ELF4 gene (Fig. 1A and 1B), which located within the the ETS domain (Fig. 1C). This amino acid site is highly conserved among the species (Table 2) and among ETS domain transcription factor super family members (Fig. 1C). Mutant ELF4 showed almost abolished activity for type I interferon trans-activation (Fig. 2E-2G), which is consistent with the susceptibility of our patient to viral and bacterial infection. Decreased CD8 T cells and NK cells (Table 1), which is consistent with findings in Elf4 knockout mice ${ }^{22}$. The hyperinflammation demonstrated as refractory oral ulcer in our patient suggests that ELF4 may negatively regulate a proinflammation pathway, in which loss of function of ELF4 results in uncontrolled inflammation. In the preparation of our manuscript, a report with similar findings was published ${ }^{23}$. Two of their three patients harbor a W251S missense mutation, the other is a frameshift mutation, all of which cause loss of function of ELF4. In our screening assay, we also found amino acid 251 is essential for type I interferon induction (Fig. 1D). These 
three patients presented with fever, oral ulcers and mucosal inflammation, but not infection. In deed they found ELF4 promotes IL1RN (an anti-inflammation gene) and limits Trem1 expression in macrophages. Our patient lacks intestinal mucosal inflammation, but presents with constipation. The phenotype discrepancy between patients with W251S and our patient needs further investigation.

In conclusion, in the present study we described a patient presenting with recurrent viral and bacterial respiratory infection, refractory oral ulcer and constipation. After a clinical diagnosis of mAID is made, we further pursued the genetic diagnosis. Whole exome sequencing found no known genes for the patient but ELF4 (chrX:129205133 A $>$ G, c.691T >C, p.W231R) is a strong candidate novel gene. We then evaluated the pathogenicity of this mutation by both various bioinformatic methods and confirmed it with experimental approach. Thus, our patient presents as a novel mAID, which we termed "X-linked autoinflammatory and immunodeficiency disease associated with ELF4, X-AIDE". More mechanistic studies are needed for more accurate understanding of this new disease.

\section{Figure legends}

Figure 1. A missense mutation in ELF4 is identified in a patient with recurrent respiratory infection and oral ulcer.

A. Sanger sequencing of the ELF4 gene in P1 and family members. Genomic DNA was used.

B. Amino acids sequence from ETS domain transcription factors family (upper) and ELF4 of different species are aligned. Some highly conserved sites are indicated.

C. Schematic diagram of the human ELF4 protein.

D. IFN- $\beta$ dual luciferase reporter screening for essential sites of ELF4 in ETS domain for induction of type I interferon (left) and western blot confirming the expression of plasmids (right). 


\section{Figure 2. W231R mutation confers ELF4 decreased stability and} trans-activation activity

A. Interactomic interactions changes predicted by DynaMut using AlphaFold2 predicted ELF4 structure. Wild-type and mutant residues are colored in light-green and are also represented as sticks alongside with the surrounding residues which are involved on any type of interactions.

B. Visual representation of $\Delta$ Vibrational Entropy Energy predicted by DynaMut using AlphaFold2 predicted ELF4 structure. Amino acids colored according to the vibrational entropy change upon mutation. BLUE represents a rigidification of the structure and RED a gain in flexibility.

C. Expression of ELF4 in PBMCs from patient (P), patient's father $(\mathrm{F})$ and patient's mother (M).

D. Protein stability test of mutant ELF4. Cycloheximide Chase Assay for W231R mutant ELF4 and WT control. Representative images from 2-3 independent experiments.

E. IFN- $\beta$ dual luciferase reporter assay for WT and mutant ELF4. hELF4, human ELF4; mELF4, mouse ELF4. Data are derived from 2-3 independent experiments.

F. IFN- $\alpha$ dual luciferase reporter assay for WT and mutant ELF4. hELF4, human ELF4; mELF4, mouse ELF4. Data are derived from 2-3 independent experiments.

G. IFN- $\beta$ dual luciferase reporter assay for the dose-effect of WT and mutant ELF4. hELF4, human ELF4; mELF4, mouse ELF4. Data are derived from 2-3 independent experiments.

Table 1. Comprehensive analysis of peripheral lymphocytes subsets

Table 2. The prediction of the identified variant in $E L F 4$ 


\section{Materials and methods}

\section{Patient}

The ethics committee of Children's Hospital of Chongqing Medical University approved the study. Blood samples from patients and unaffected relatives were collected for molecular studies, which were performed in accordance with the Declaration of Helsinki.

\section{Patient consent statement}

Written informed consent for participation in the study and for the publication of the research work was obtained from patients' parents.

\section{Genetic Analyses}

Genomic DNA were isolated from peripheral blood samples. Disease-causing mutations were screened using whole genome sequencing (WES) (MyGenostics, Inc.). Candidate mutations were confirmed by Sanger sequencing using the following primers: seq F: 5'- AAGACCAAGGGCAACCGAAG -3', seq R: 5'-CCATGAAAGCGAGGAGGACT -3'.

\section{Construction of plasmids}

7.1-pCMV-3×Flag wild type human and mouse ELF4 plasmids were as previously described $^{8}$. W231R mutant of human ELF4 and W230R mutant of mouse ELF4 were generated by overlap PCR method using WT as template. Mutations were confirmed by Sanger sequencing.

\section{Cells}

HEK 293T (Human Embryonic Kidney 293 cells transformed by expression of the large T antigen from SV40) (ATCC, CRL-11268) were cultured in Dulbecco's modified Eagle medium (DMEM).

\section{Western blotting}

Cells were harvested and lysed in RIPA buffer (Beyotime, P0013C), containing 
complete mini protease inhibitor cocktail (Roche, 04693124001). Proteins were separated by SDS-PAGE and transferred to polyvinylidene fluoride membranes (GE, PVDF 0.45UM, 10600023). Non-specific binding was blocked using 5\% non-fat milk and the primary antibody (anti-Flag, Sigma F3165, anti-GAPDH, Invitrogen MA1-16757, anti-ELF4, Sigma AV38028 was incubated at $4^{\circ} \mathrm{C}$ overnight, the secondary antibody was incubated at room temperature for $1 \mathrm{~h}$. Signals were visualized by chemiluminescence (Millipore Corporation, Billerica, MA, USA).

\section{Cycloheximide Chase Assay}

This assay is adapted from previous report ${ }^{24}$. Briefly, HEK293T cells were plated and transfected with various WT and mutant 7.1-pCMV-3×Flag expression plasmids. 24 hours later, cycloheximide was added $(1 \mathrm{ug} / \mathrm{ml})$ and cells were collected at different time point for western blot detection.

\section{Statistical analysis}

Samples were compared using two-tailed, unpaired Student's $t$-test with GraphPad Prism 7.00. Error bars were represented by SEM. ${ }^{*} P<0.05$, $* * P<0.01$, $* * * P<$ 0.001 .

\section{Authors' contributions}

HQ.D and XD.Z conceived and supervised the project, reviewed and revised the manuscript; HQ.D designed the experiments, analyzed data and wrote the manuscript. G.S performed the experiments, analyzed data and followed the patient. HW.X and LL.C provided experimental expertise. FP.Y provided scientific sights in designing the project and revising the manuscript. Others provided essential help for clinical management and follow-up of the patient and valuable clinical expertise in reviewing the manuscript.

\section{Acknowledgement}


We thank the patient and his family for their cooperation, especially for the patient's mother whose perseverance in caring for her child gives us strength to pursue a definitive genetic diagnosis and next a more effective personalized treatment.

\section{Fundings}

Sanming Project of Medicine in Shenzhen (SZSM201812002);

Livelihood Project of Chongqing, China (cstc2018jscxmsybX0005)

National Natural Science Foundation of China (81974255)

\section{Data availability}

The data that support the findings of this study are available from the corresponding authors upon reasonable request.

\section{Conflict of interest}

No conflict of interest is declared by the authors.

\section{Author consent}

We confirm that the manuscript has been read and approved by all named authors.

\section{Ethics approval}

The ethics committee of Children's Hospital of Chongqing Medical University approved the study.

Blood samples collection were performed in accordance with the Declaration of Helsinki.

\section{References}

1. Martinon, F. \& Aksentijevich, I. New players driving inflammation in monogenic autoinflammatory diseases. Nat Rev Rheumatol 11, 11-20 (2015); Park, H., Bourla, A.B., Kastner, D.L., Colbert, R.A. 
\& Siegel, R.M. Lighting the fires within: the cell biology of autoinflammatory diseases. Nat Rev Immunol 12, 570-580 (2012).

2. Nigrovic, P.A., Lee, P.Y. \& Hoffman, H.M. Monogenic autoinflammatory disorders: Conceptual overview, phenotype, and clinical approach. J Allergy Clin Immunol 146, 925-937 (2020).

3. Hashkes, P.J., Barron, K.S. \& Laxer, R.M. Clinical approach to the diagnosis of autoinflammatory diseases. Textbook of autoinflammation. Springer, 2019, pp 203-223.

4. Gattorno, M. et al. A diagnostic score for molecular analysis of hereditary autoinflammatory syndromes with periodic fever in children. Arthritis Rheum 58, 1823-1832 (2008).

5. Touitou, I. \& Aksentijevich, I. Genetic approach to the diagnosis of autoinflammatory diseases. Textbook of Autoinflammation. Springer, 2019, pp 225-237.

6. Ombrello, M.J. Monogenic Autoinflammatory Diseases Associated with Immunodeficiency. Textbook of Autoinflammation. Springer, 2019, pp 499-514.

7. Suico, M.A., Shuto, T. \& Kai, H. Roles and regulations of the ETS transcription factor ELF4/MEF. J Mol Cell Biol 9, 168-177 (2017).

8. You, F. et al. ELF4 is critical for induction of type I interferon and the host antiviral response. Nat Immunol 14, 1237-1246 (2013).

9. Du, H. et al. Suppression of ELF4 in ulcerative colitis predisposes 
host to colorectal cancer. 102169 (2021).

10. Ding, Y. et al. Reference values for peripheral blood lymphocyte subsets of healthy children in China. J Allergy Clin Immunol 142, 970-973 e978 (2018).

11. Takeda, J.I. et al. InMeRF: prediction of pathogenicity of missense variants by individual modeling for each amino acid substitution. NAR Genom Bioinform 2, lqaa038 (2020).

12. Sharrocks, A.D. The ETS-domain transcription factor family. Nat Rev Mol Cell Biol 2, 827-837 (2001).

13. Rodrigues, C.H., Pires, D.E. \& Ascher, D.B. DynaMut: predicting the impact of mutations on protein conformation, flexibility and stability. Nucleic Acids Res 46, W350-W355 (2018).

14. Jumper, J. et al. Highly accurate protein structure prediction with AlphaFold. Nature (2021).

15. Reva, B., Antipin, Y. \& Sander, C. Predicting the functional impact of protein mutations: application to cancer genomics. Nucleic Acids Res 39, e118 (2011).

16. McDermott, M.F. et al. Germline mutations in the extracellular domains of the $55 \mathrm{kDa}$ TNF receptor, TNFR1, define a family of dominantly inherited autoinflammatory syndromes. Cell 97, 133-144 (1999).

17. Zeidan, M.J. et al. Behcet's disease physiopathology: a 
contemporary review. Auto Immun Highlights 7, 4 (2016); Zhou, Q. et al. Loss-of-function mutations in TNFAIP3 leading to A20 haploinsufficiency cause an early-onset autoinflammatory disease. Nat Genet 48, 67-73 (2016).

18. Martin-Nalda, A. et al. Severe Autoinflammatory Manifestations and Antibody Deficiency Due to Novel Hypermorphic PLCG2 Mutations. J Clin Immunol 40, 987-1000 (2020).

19. Zinngrebe, J. et al. --LUBAC deficiency perturbs TLR3 signaling to cause immunodeficiency and autoinflammation. J Exp Med 213, 2671-2689 (2016).

20. Chakraborty, P.K. et al. Mutations in TRNT1 cause congenital sideroblastic anemia with immunodeficiency, fevers, and developmental delay (SIFD). Blood 124, 2867-2871 (2014).

21. Standing, A.S. et al. Autoinflammatory periodic fever, immunodeficiency, and thrombocytopenia (PFIT) caused by mutation in actin-regulatory gene WDR1. $J$ Exp Med 214, 59-71 (2017).

22. Lacorazza, H.D. et al. The ETS protein MEF plays a critical role in perforin gene expression and the development of natural killer and NK-T cells. Immunity 17, 437-449 (2002).

23. Tyler, P.M. et al. Human autoinflammatory disease reveals ELF4 as a transcriptional regulator of inflammation. Nat Immunol (2021). 
medRxiv preprint doi: https://doi.org/10.1101/2021.09.22.21263689; this version posted September 25, 2021. The copyright holder for this preprint (which was not certified by peer review) is the author/funder, who has granted medRxiv a license to display the preprint in perpetuity.

It is made available under a CC-BY-ND 4.0 International license .

24. Kao, S.H. et al. Analysis of Protein Stability by the Cycloheximide

Chase Assay. Bio Protoc 5 (2015). 
medRxiv preprint doi: https://doi.org/10.1101/2021.09.22.21263689; this version posted September 25, 2021. The copyright holder for this preprint (which was not certified by peer review) is the author/funder, who has granted medRxiv a license to display the preprint in perpetuity.

$\mathrm{F}$

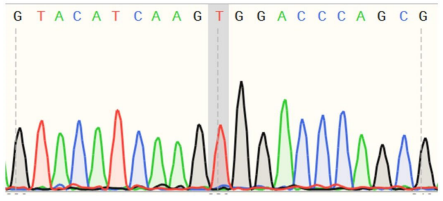

It is madelefyailable under a CC-BY-ND 4.0 InternaßBnal license .

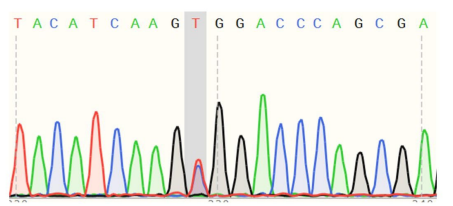

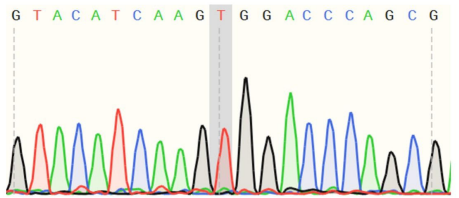

$\mathrm{P}$

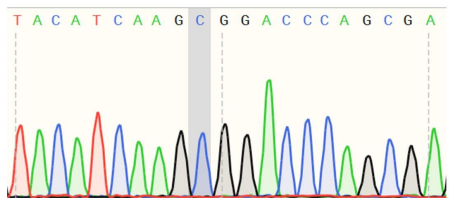

B.

211\&212 219

231

239

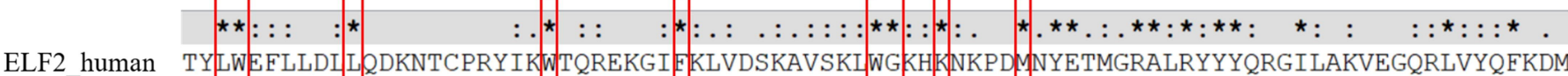

$251 \& 252$

260

\section{5} ELF4_human IYUWEFLLAIILDRNTCPKYIKWTQREKGI EKLVDSKAVSKIWGKQ KNKPDMNYETMGRALRYYYQRGILAKVEGQRLVYQFKEM ELF1_human IYLWEFLLAI IDDKATCPKYIKWTQREKGI EKLVDSKAVSRIWGKHKNKPDMNYETMGRALRYYYQRGILAKVEGQRLVYQFKEM ETV6_human RLIWDYVYQI L SDS-RYENF IRWEDKESKI ERIVDPNGLARIWGNHKNRTNMTYEKMSRALRHYYKLNI IRKEPGQRLLFRFMKT ETV7 human RLLWDYVYQILLDT-RYEPY IKWEDKDAKI ERVVDPNGLARIWGNHUNRVNMTYEKMSRALRHYYKLNI IKKEPGQKLLFRFLKT ELF5 human SHLWEFVRDILLSPEENCGILEWEDREQGI ERVVKSEALAKMWGRRKKNDRMTYEKLSRALRYYYKTGILERVD-RRLVYKFGKN ELF3_human THLWEFIRDII IHPELNEGLMKWENRHEGVEKELRSEAVAQIWGRKKKNSNMTYEKLSRAMRYYYKREILERVDGRRLVYKFGKN EHF_human THLWEFIRDI ILNPDKNPGLIKWEDRSEGV ERFLKSEAVAQI WGKKKKNNSSMTYEKLSRAMRYYYKREILERVDGRRLVYKFGKN

\section{1}

M.Musculus

R.Norvegicus

B.Taurus

YLWEFLLALLQDRNTCPKYIKWTQREKGIFKLVDSKAVSKLWGKQKNKPDMNYETMGRALRYYYQRGILA YLWEFLLALLQDRNTCPKYIKWTQREKGIFKLVDSKAVSKLWGKQKNKPDMNYETMGRALRYYYQRGILA YLWEFLLALLQDRNTCPKY IKWTQREKGIFKLVDSKAVSKLWGKQKNKPDMNYETMGRALRYYYQRGILA C.lupus familiaris YLWEFLLALLQDRNTCPKYIKWTQREKGIFKLVDSKAVSKLWGKQKNKPDMNYETMGRALRYYYQRGILA H.Sapiens M.Mulatta YLWEFLLALLQDRNTCPKYIKWTQREKGIFKLVDSKAVSKLWGKQKNKPDMNYETMGRALRYYYQRGILA YLWEFLLALLQDRNTCPKYIKWTQREKGIFKLVDSKAVSKLWGKQKNKPDMNYETMGRALRYYYQRGILA

E.caballus YLWEFLLALLQDRNTCPKYIKWTQREKGIFKLVDSKAVSKLWGKQKNKPDMNYETMGRALRYYYQRGILA

S.scrofa

C.

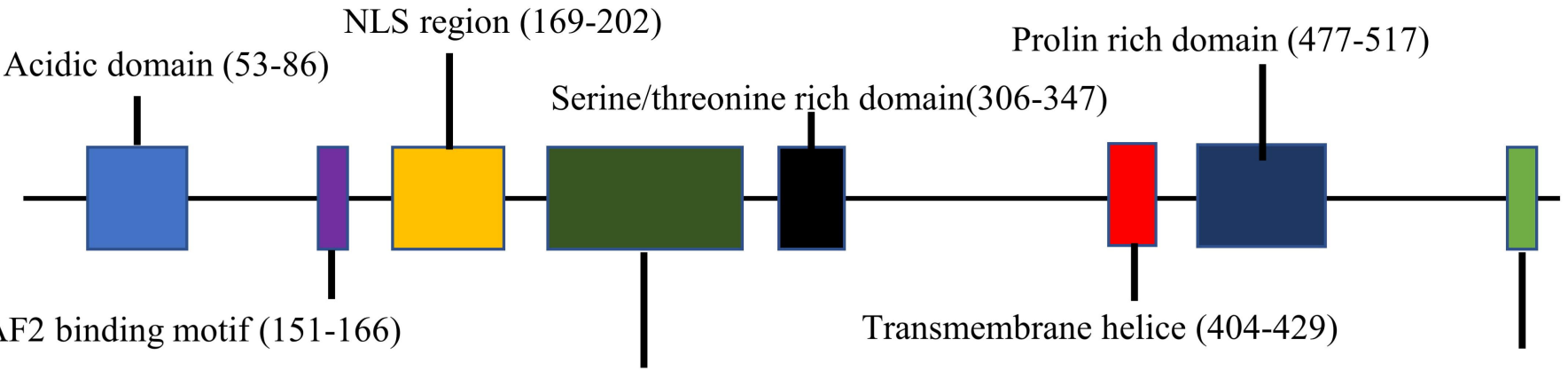

ETS domain (207-291)

Motif for sumoylation (656-659)

D.
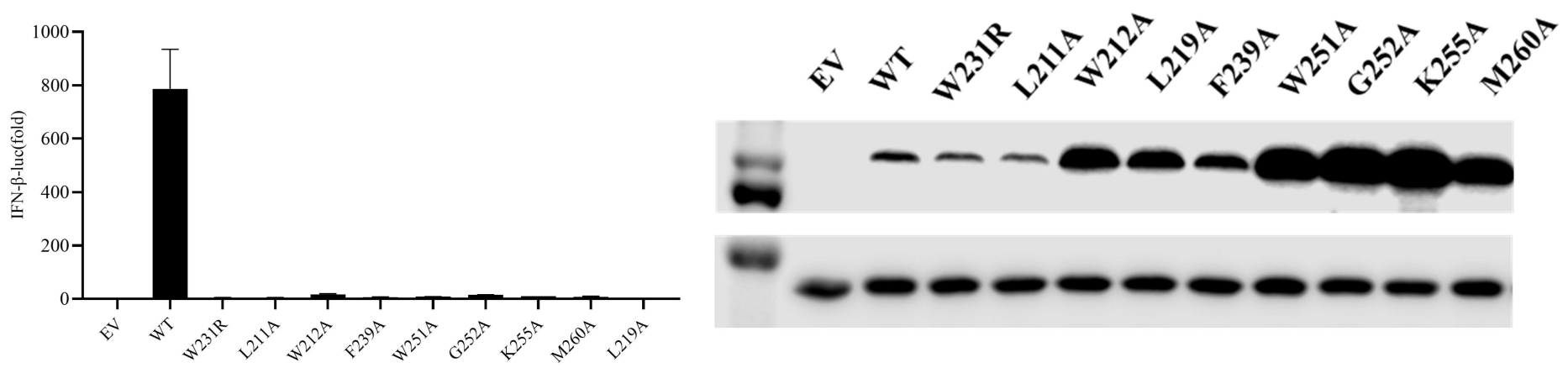
medRxiv preprint doi: https://doi.org/10.1101/2021.09.22.21263689; this versioinposted \$eptember 25 2021. Tige cppyright holderfor this preprint (which was not certified by peer review) is the author/funder, who has granted medRxiv a license to display the preprint in perpetuity.

WT
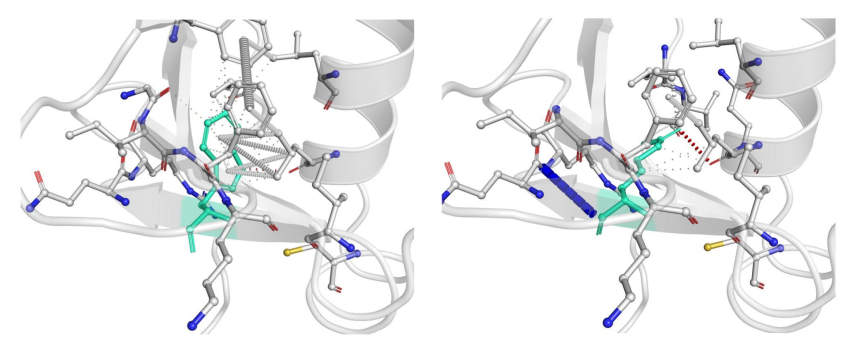

C.

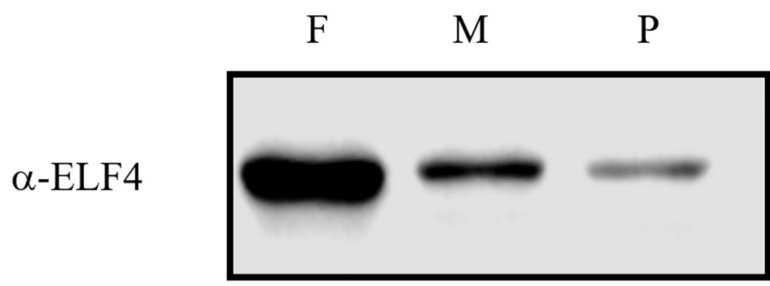

$\alpha-G A P D H$

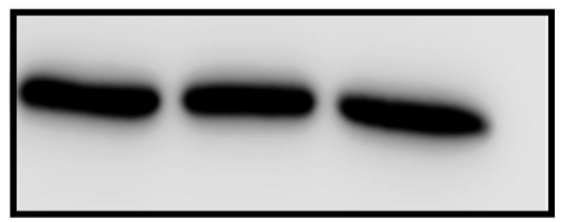

E.

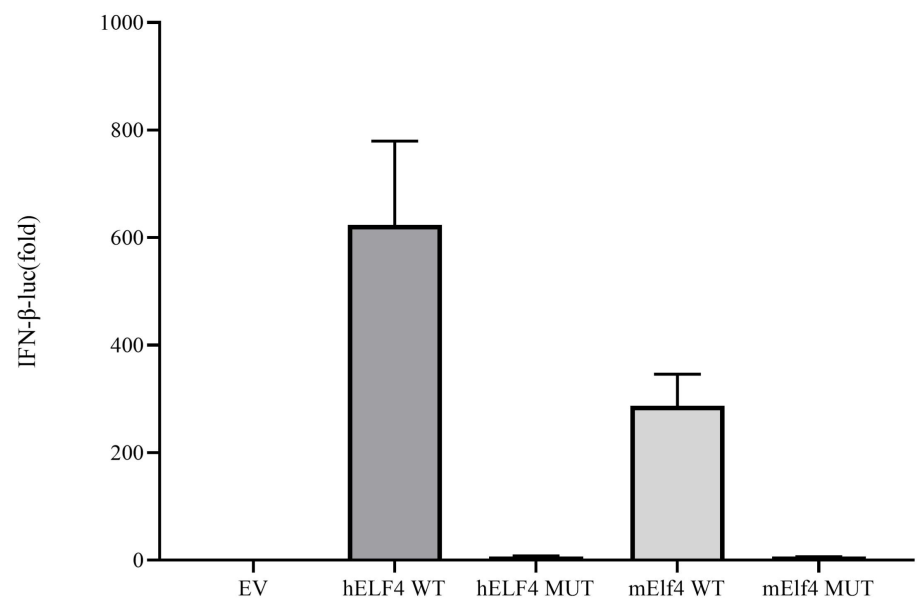

D.
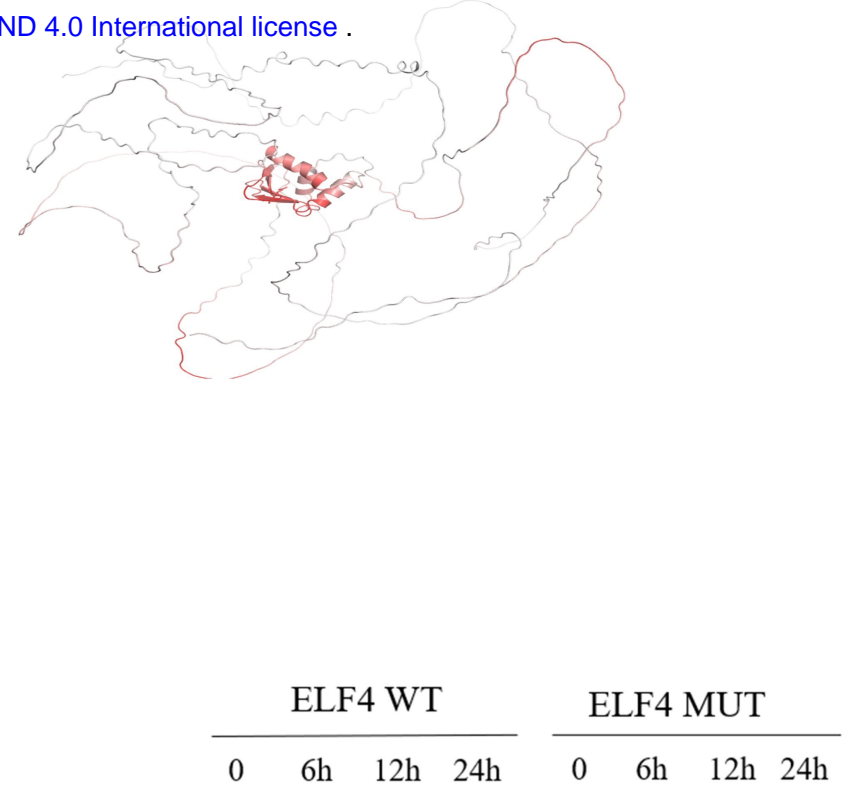

$\alpha-$ FLAG

$\alpha-$ GAPDH

F.

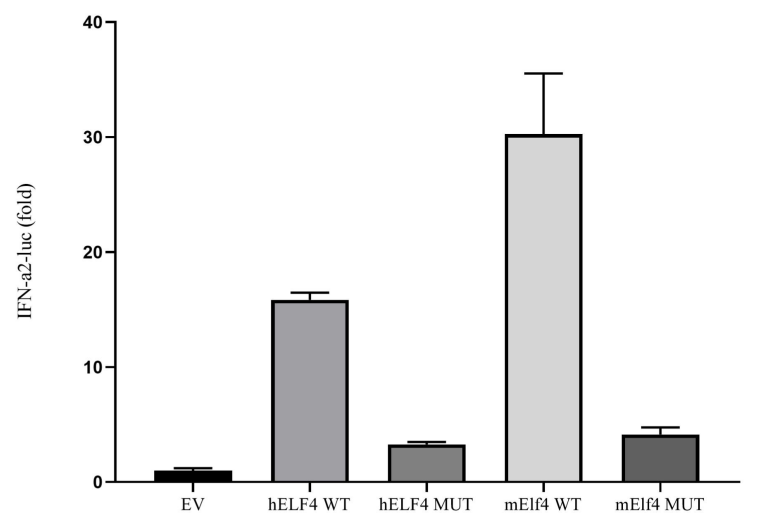

G.

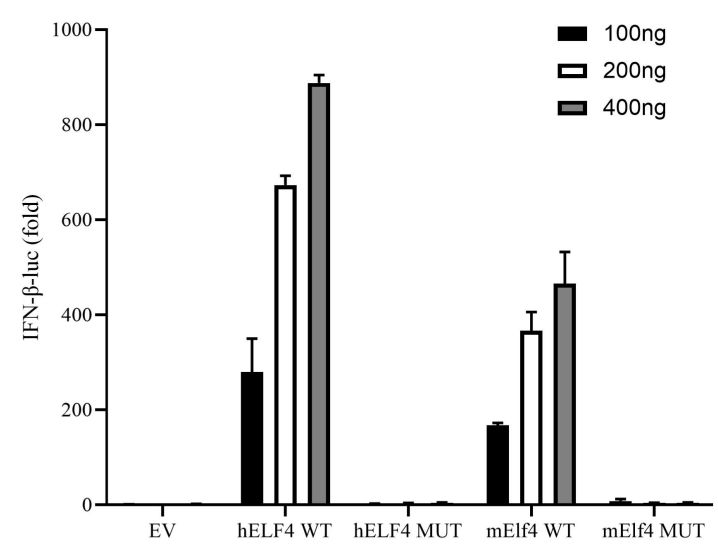

